\title{
Assembly Line Balancing Using Heuristic Methods
}

\section{${ }^{1}$ E.J. Panggabean, ${ }^{2}$ M.R.B. Barjaman and ${ }^{3}$ S.P.A. Nugraha}

${ }^{1}$ School of Industrial and System Engineering, Telkom University, Bandung, Indonesia

${ }^{2}$ School of Industrial and System Engineering, Telkom University, Bandung, Indonesia

${ }^{3}$ School of Industrial and System Engineering, Telkom University, Bandung, Indonesia

Correspondence Author: E.J. Panggabean, School of Industrial and System Engineering, Telkom University, Bandung, Indonesia.

Received date: 11 April 2018, Accepted date: 25 July 2018, Online date: 8 August 2018

Copyright: (C) 2018 G. Naveen Balajiet al. This is an open-access article distributed under the terms of the Creative Commons Attribution License, which permits unrestricted use, distribution, and reproduction in any medium, provided the original author and source are credited.

\begin{abstract}
The assembly line balancing is the most essential parts for many kinds of manufacturing industries which required to control and supervise carefully. The success of achieving the goals of production is influenced significantly by assembly line balancing. Most of manufacturers and researchers make a try to look for the best methods to keep the assembly line in production floor stay balanced. This research is done to help the company especially in the process of assembly line in Final Area of BMW F30 Series 3 which for improving the productivity in the assembly line particularly to increase line efficiency and decrease smoothness index. The authors consider three heuristics methods which almost lead to the reduction of the number of workstations. This paper shows the application of assembly line balancing methods. The methods are Largest Candidate Rule (LCR), Kilbridge and Wester (KWC), and Ranked Positional Weight (RPW) methods. The result of those methods is compared to find out the best line efficiency, smoothness index and the lowest balance delay.
\end{abstract}

Key words: Assembly Line Balancing ${ }^{\mathrm{LCR}} \bullet \mathrm{KWM} \bullet \mathrm{RPW} \bullet$ Line Efficiency.

\section{INTRODUCTION}

In general, a production planning is a complete specification of the amounts of each end item or final product and subassembly produced, the exact timing of the production lot size, and the final schedule of completion (Nahmias, 2009, p367). A typical large manufacturing business that's engaging production planning will aim to maximize profitability while maintaining a satisfied consumer base. The objective of production planning is to provide appropriates finished goods by period to meet the sales plan. A company engaged in the manufacturing industry has a production floor that is used as a production system. Production system is a set of interacting subsystems for transforming inputs into production outputs staying within financial and production capacity constraints. The assembly line balancing is the most essential parts for many kinds of manufacturing industries which required to control and supervise carefully. The success of achieving the goals of production is influenced significantly by assembly line balancing. Most of manufacturers and researchers make a try to look for the best methods to keep the assembly line in production floor stay balanced. While the goal of assembly line balancing namely (Gasperz, 2004): 1) Balancing the workload allocated to each workstation so that each workstation is completed at a balanced time. 2) Prevent bottleneck (an operation that limits output and production frequency). 3) Keep the assembly crossing smoothly and continuously. 4) Increase efficiency or productivity. This research is applying the three heuristic methods to learn about the process of assembly line in Final Area of BMW F30 Series 3. In this paper, those three assembly line balancing methods were Largest Candidate Rule (LCR), Kilbridge and Wester (KWC), and Ranked Positional Weight (RPW) which will be compared and selected as the best one for assembly line of Final Area.

\section{Assembly Line Balancing:}

An assembly system consists of workstations where some specific tasks are carried to produce a product. Work stations are linked together by a transportation system. Transportation system moves work in process from work station to work station. Work stations together with transportation system is called assembly line. Simple assembly line balancing problems are classified into two types, type I and type II. In type I problems, the required production rate (i.e. cycle time), assembly tasks, tasks times, and precedence requirements is given. The objective of this is to minimize the number of workstations. A line with fewer stations results in lower labor costs and reduced space requirements. Type I problems generally occurs at the time of designing new assembly lines. To achieve the forecast demand the number of workstations should be reduced. For expansion (when demand is increased) type I problems also can be used to minimize the number of extra stations need to install. In type II problems, when the number of work stations or number of employees is fixed, the objective is to minimize the cycle time. This leads to maximize the production rate. Type II balancing problems generally occurs, when the organization wants to produce the optimum number of items by using a fixed number work stations without expansion. In this type it is necessary to identify precedence, and constraints. While balancing the main line, it is necessary to consider subassembly lines. Type I problems are more common than type II. Each workstation process tasks assigned to it within cycle time. At the end of each cycle a product comes out of the last station. Tasks cannot be assigned to stations arbitrarily. There are some technological constraints such that some tasks should has processed before some specific task set, i.e. tasks have some precedence relations. These precedence relationships can be shown on a precedence network. Heuristic Methods of Line Balancing:

1. Moodie -Young Method

2. Killbridge and Wester Heuristic

3. Hoffmans or Precedence Matrix

4. Immediate Update First Fit Method

5. Ranked Position Weighted Method (RPW) 
When designing an assembly line the following restrictions must be imposed on grouping of workelements.1) Precedence relationship.2) The number of work elements cannot be greater than the number of workstation. The minimum number of workstations is one.3) The cycle time is greater than or equal to the maximum of any station time and of the time of any work elements. The station time should not be exceeding the cycle time.

\section{Case Study:}

PT Gaya Motor is the plant which focuses on the assembly process of some major vehicles such as BMW, Toyota, Daihatsu, Suzuki and etc. Services in assembling the vehicle consists of welding, painting, and packing. This plant is utilizing the production process by using the system of Make to Order since their assembly process starts only after a customer's order is received. The details of the existing assembly line for Final Area of BMW F30 Series 3 are given in Table 1 and 2. The present production rate is 40 units per day. The company want to increase the line efficiency and to decrease the bottleneck. So, it is decided to balance the assembly line.

Existing Assembly Line:

Table 1: Work elements time and precedence at present.

\begin{tabular}{|c|c|l|c|}
\hline \multicolumn{2}{|c|}{ Operation } & \multicolumn{1}{|c|}{ Time } & Workstation No \\
\hline 1 & 5 & $504 \sec (8.24 \mathrm{~min})$ & F01 \\
\hline 6 & 12 & $776 \mathrm{sec}(12.56 \mathrm{~min})$ & F02 \\
\hline 13 & 21 & $1349 \sec (22.29 \mathrm{~min})$ & F03 \\
\hline 22 & 26 & $1034 \sec (17.14 \mathrm{~min})$ & F04 \\
\hline 27 & 37 & $1098 \sec (18.18 \mathrm{~min})$ & F05 \\
\hline
\end{tabular}

Table 2: Existing work elements time and precedence.

\begin{tabular}{|c|c|c|}
\hline No.Operation & Time (sec) & Predecessor \\
\hline 1 & 21 & - \\
\hline 2 & 68 & - \\
\hline 3 & 197 & 2 \\
\hline 4 & 73 & 3 \\
\hline 5 & 145 & - \\
\hline 6 & 71 & 5 \\
\hline 7 & 151 & 6 \\
\hline 8 & 74 & 3,4 \\
\hline 9 & 85 & 3 \\
\hline 10 & 185 & - \\
\hline 11 & 149 & 2,9 \\
\hline 12 & 61 & 11 \\
\hline 13 & 128 & 10,12 \\
\hline 14 & 201 & - \\
\hline 15 & 150 & 7,14 \\
\hline 16 & 300 & 12,13 \\
\hline 17 & 165 & 15,16 \\
\hline 18 & 61 & 10,17 \\
\hline 19 & 86 & - \\
\hline 20 & 1.2 & 19 \\
\hline 21 & 257 & 20 \\
\hline 22 & 93 & 21 \\
\hline 23 & 187 & 20,21 \\
\hline 24 & 269 & 22,23 \\
\hline 25 & 76 & 24 \\
\hline 26 & 152 & 24 \\
\hline 27 & 70 & 24 \\
\hline 28 & 96 & 27,25 \\
\hline 29 & 69 & 20,26 \\
\hline 30 & 77 & 29 \\
\hline 31 & 78 & 28,30 \\
\hline 32 & 61 & 31 \\
\hline 33 & 96 & 32 \\
\hline 34 & 125 & 18,30 \\
\hline 35 & 95 & 34 \\
\hline 36 & 90 & 35 \\
\hline 37 & 241 & 35,36 \\
\hline
\end{tabular}

\section{Heuristic Methods:}

This study is to find a task-workstation assignment solution that balances the processing times among workstations procedures use Large Candidate Rule, Kilbridge and Wester's and Ranked Positional Weight method to generate an initial solution, and a task reassignment algorithm to improve the initial solution by reassigning tasks to new workstations for increasing the line efficiency and decrease the smoothness index.

\section{Largest Candidate Rule (LCR) Method:}

In this approach work elements were arranged in descending order according to their Operation Time as presented in Tables 4 and 5. Worker was assigned by elements at the first workstation by beginning at the top of the list and selected the first element that meets precedence requirements and does not cause the total sum of Time at that station to exceed the Cycle Time when an element is selected for assignment to the station, started backward at the top of the list for the subsequent assignments. The procedure then followed by stating that no more elements could be assigned without exceeding Cycle Time and proceeded to the next station. Consequently, repeating the previous steps for as many additional stations as possible until all elements have been assigned as shown in the precedence diagram. 


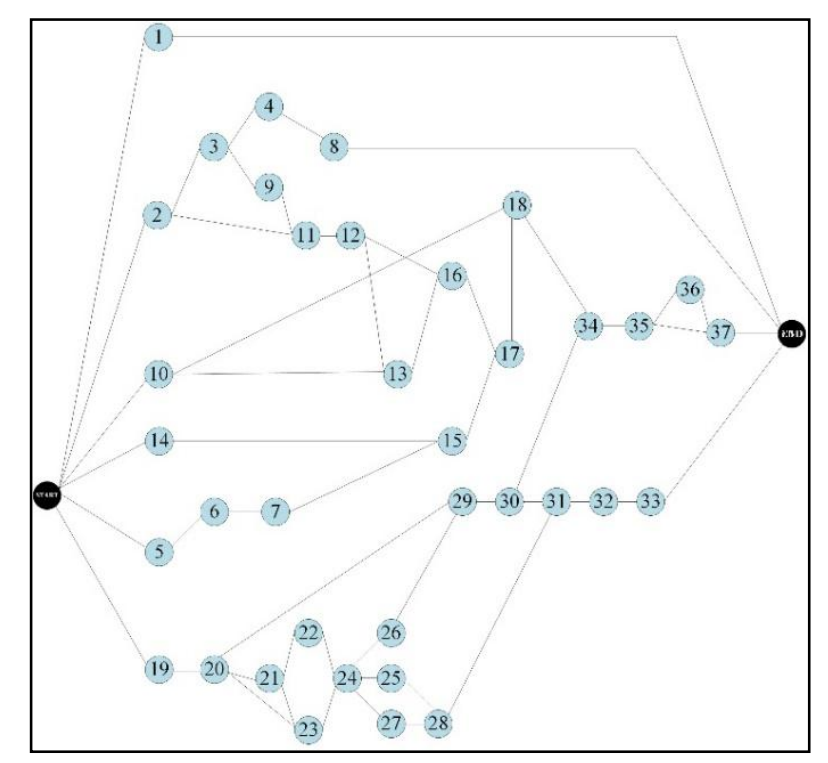

Fig. 1: Precedence Diagram.

Calculation of Existing Assembly Line

No of operation $=37$

Cycle time $(\mathrm{C})=23.69 \mathrm{~min} / 1449 \mathrm{sec}$

Workstation amount $=5$

Demand rate $=1500 /$ year

Assembly line operation time $=630$ hour/year

Sum of station time $(\mathrm{STi})=79.21 \mathrm{~min} / 4761 \mathrm{sec}$

Table 3: Existing assembly line calculation.

\begin{tabular}{|c|c|l|c|c|c|}
\hline \multicolumn{2}{|c|}{ Operation } & ST & Stmax (min) & $\begin{array}{c}\text { Idle Time } \\
(\mathbf{m i n})\end{array}$ & Idle $^{\mathbf{2}}$ \\
\hline 1 & 5 & $504 \sec (8.24 \mathrm{~min})$ & 22.29 & 14.05 & 197.403 \\
\hline 6 & 12 & $776 \mathrm{sec}(12.56 \mathrm{~min})$ & 22.29 & 9.73 & 94.673 \\
\hline 13 & 21 & $1349 \sec (22.29 \mathrm{~min})$ & 22.29 & 0.00 & 0.000 \\
\hline 22 & 26 & $1034 \sec (17.14 \mathrm{~min})$ & 22.29 & 5.15 & 26.523 \\
\hline 27 & 37 & $1098 \sec (18.18 \mathrm{~min})$ & 22.29 & 4.11 & 16.892 \\
\hline \multicolumn{2}{|l|}{$\boldsymbol{\Sigma}$ (total) } & $\mathbf{7 9 . 2 1}$ & $\mathbf{1 1 1 . 4 5}$ & $\mathbf{3 3 . 0 4}$ & $\mathbf{3 3 5 . 4 9}$ \\
\hline
\end{tabular}

$L E$ (Line efficiency) $=\frac{\sum S T}{\sum S T \max } \times 100 \%$
$S I($ Smoothing Index $)=\sqrt{\sum(S T \max -S T)^{2}}$

Calculation of Existing Assembly Line

No of operation $=37$

Cycle time $(C)=23.69 \mathrm{~min} / 1449 \mathrm{sec}$

Demand Rate $=1550 /$ year

Assembly line operation time $=630$ hour/year

Sum of station time $(\mathrm{STi})=79.21 \mathrm{~min} / 4761 \mathrm{sec}$

Minimum No.Workstation (Kmin):

$\sum S T i$

Cycle Time

$\mathrm{Kmin}=\quad=79.21 / 23.69=3.34(4 \mathrm{WS})$

\begin{tabular}{|c|c|c|c|}
\hline $\begin{array}{c}\text { Workstation I } \\
14,2,3,10,5,19,9, \\
4,8,6\end{array}$ & $\begin{array}{c}\text { Workstation II } \\
7,11,12,13,16,15,15 \\
17,18\end{array}$ & $\begin{array}{c}\text { Workstation III } \\
20,21,23,22,24, \\
26,25,27\end{array}$ & $\begin{array}{c}\text { Workstation IV } \\
29,30,34,35,36,37, \\
28,31,32,33,1\end{array}$ \\
\cline { 3 - 4 }
\end{tabular}

Fig. 2: Configuration Layout for Assembly Line Following LCR. 
Citation: E.J. Panggabean, M.R.B. Barjaman and.P.A. Nugraha, 2018. Assembly Line Balancing Using Heuristic Methods. Advances in Natural and Applied Sciences., 12(8): 1-8.DOI: 10.22587/anas.2018.12.8.1

Table 4: Work element arranged in descending order

\begin{tabular}{|c|c|c|}
\hline No.Ope ration & Time (sec) & Predecessor \\
\hline 16 & 300 & 12,13 \\
\hline 24 & 269 & 22,23 \\
\hline 21 & 257 & 20 \\
\hline 37 & 241 & 35,36 \\
\hline 14 & 201 & - \\
\hline 3 & 197 & 2 \\
\hline 23 & 187 & 20,21 \\
\hline 10 & 185 & - \\
\hline 17 & 165 & 15,16 \\
\hline 26 & 152 & 24 \\
\hline 7 & 151 & 6 \\
\hline 15 & 150 & 7,14 \\
\hline 11 & 149 & 2,9 \\
\hline 5 & 145 & - \\
\hline 13 & 128 & 10,12 \\
\hline 34 & 125 & 18,30 \\
\hline 28 & 96 & 27,25 \\
\hline 33 & 96 & 32 \\
\hline 35 & 95 & 34 \\
\hline 22 & 93 & 21 \\
\hline 36 & 90 & 35 \\
\hline 19 & 86 & - \\
\hline 9 & 85 & 3 \\
\hline 31 & 78 & 28,30 \\
\hline 30 & 77 & 29 \\
\hline 25 & 76 & 24 \\
\hline 8 & 74 & 3,4 \\
\hline 4 & 73 & 3 \\
\hline 6 & 71 & 5 \\
\hline 27 & 70 & 24 \\
\hline 29 & 69 & 20,26 \\
\hline 2 & 68 & - \\
\hline 12 & 61 & 11 \\
\hline 18 & 61 & 10,17 \\
\hline 32 & 61 & 31 \\
\hline 1 & 21 & - \\
\hline 20 & 1.2 & 19 \\
\hline
\end{tabular}

$$
\begin{aligned}
& L E(\text { Line efficiency })=\frac{\sum S T}{\sum S T \max } \times 100 \% \\
& S I(\text { Smoothing Index })=\sqrt{\sum(S T \max -S T)^{2}} \\
&
\end{aligned}
$$

Kilbridge And Wester (KWC) Method:

Work elements in this method are selected for assignment to stations according to their position in the precedence diagram. Work elements were arranged into columns, and then organized into a list according to their column, with the elements in the first column listed first. Table 7 shows a list of elements of the column method, starting with the highest value of the time for each column. Consequently, repeating the previous steps for as many additional stations as possible until all elements have been assigned as shown in the precedence diagram Fig 3.

Table 6: LCR Method Assembly Line Calculation.

Table 6: LCR Method Assembly Line Calculation.
\begin{tabular}{|c|c|c|c|c|}
\hline Workstaion & ST (sec) & Stmax (sec) & Idle Time & Idle $^{\mathbf{2}}$ \\
\hline I & 1185 & 1185 & 0.00 & 0.000 \\
\hline II & 1165 & 1185 & 20.00 & 400.000 \\
\hline III & 1105.2 & 1185 & 79.80 & 6368.040 \\
\hline IV & 1049 & 1185 & 136.00 & 18496.000 \\
\hline $\mathbf{\text { (total) }}$ & $\mathbf{4 5 0 4 . 2 0}$ & $\mathbf{4 7 4 0 . 0 0}$ & $\mathbf{2 3 5 . 8 0}$ & $\mathbf{2 5 2 6 4 . 0 4}$ \\
\hline
\end{tabular}

Line Efficiency $($ LE) $=(74.24 / 77.80) * 100 \%=\mathbf{9 5 , 4 2} \%$

Smoothing Index $=\sqrt{ } 335.49=\mathbf{2 . 4 7}$

Balance Delay $=100 \%-\mathrm{LE}=\mathbf{4 . 5 8} \%$
Table 5: Work element arranged according to time values for LCR

\begin{tabular}{|c|c|c|c|c|}
\hline No.Operation & Time (sec) & Predecessor & Station no & Station Time \\
\hline 14 & 201 & - & \multirow{10}{*}{ I } & \multirow{10}{*}{$\begin{array}{c}1185 \mathrm{sec} \\
(19.45 \mathrm{~min})\end{array}$} \\
\hline 2 & 68 & - & & \\
\hline 3 & 197 & 2 & & \\
\hline 10 & 185 & - & & \\
\hline 5 & 145 & - & & \\
\hline 19 & 86 & - & & \\
\hline 9 & 85 & 3 & & \\
\hline 4 & 73 & 3 & & \\
\hline 8 & 74 & 3,4 & & \\
\hline 6 & 71 & 5 & & \\
\hline 7 & 151 & 6 & \multirow{8}{*}{ II } & \multirow{8}{*}{$\begin{array}{c}1165 \mathrm{sec} \\
(19.25 \mathrm{~min})\end{array}$} \\
\hline 11 & 149 & 2,9 & & \\
\hline 12 & 61 & 11 & & \\
\hline 13 & 128 & 10,12 & & \\
\hline 16 & 300 & 12,13 & & \\
\hline 15 & 150 & 7,14 & & \\
\hline 17 & 165 & 15,16 & & \\
\hline 18 & 61 & 10,17 & & \\
\hline 20 & 1.2 & 19 & \multirow{8}{*}{ III } & \multirow{8}{*}{$\begin{array}{c}1105.2 \mathrm{sec} \\
(18.25 \mathrm{~min})\end{array}$} \\
\hline 21 & 257 & 20 & & \\
\hline 23 & 187 & 20,21 & & \\
\hline 22 & 93 & 21 & & \\
\hline 24 & 269 & 22,23 & & \\
\hline 26 & 152 & 24 & & \\
\hline 25 & 76 & 24 & & \\
\hline 27 & 70 & 24 & & \\
\hline 29 & 69 & 20,26 & \multirow{11}{*}{ IV } & \multirow{11}{*}{$\begin{array}{c}1049 \mathrm{sec} \\
(17.29 \mathrm{~min})\end{array}$} \\
\hline 30 & 77 & 29 & & \\
\hline 34 & 125 & 18,30 & & \\
\hline 35 & 95 & 34 & & \\
\hline 36 & 90 & 35 & & \\
\hline 37 & 241 & 35,36 & & \\
\hline 28 & 96 & 27,25 & & \\
\hline 31 & 78 & 28,30 & & \\
\hline 32 & 61 & 31 & & \\
\hline 33 & 96 & 32 & & \\
\hline 1 & 21 & - & & \\
\hline
\end{tabular}




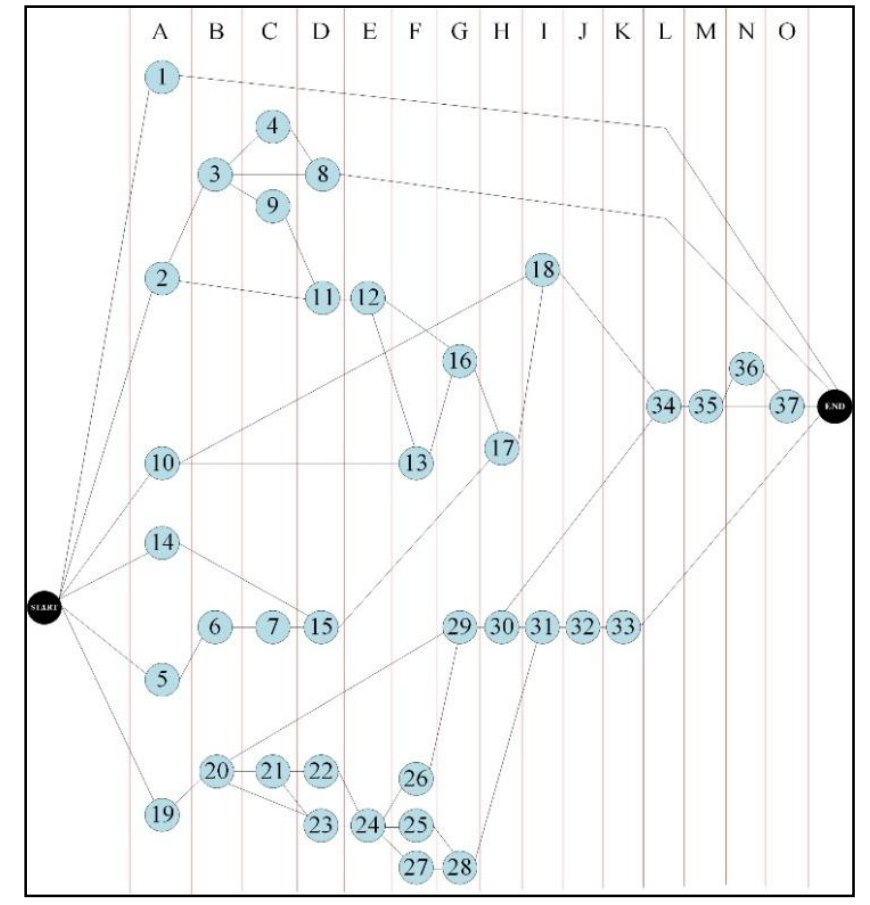

Fig. 3: Work Element Arranged into Column for the KWM.

Table 7: Work element assigned according to column method. 
Citation: E.J. Panggabean, M.R.B. Barjaman and.P.A. Nugraha, 2018. Assembly Line Balancing Using Heuristic Methods. Advances in Natural and Applied Sciences., 12(8): 1-8.DOI: 10.22587/anas.2018.12.8.1

\begin{tabular}{|c|c|c|c|}
\hline No.Operation & Time (sec) & Column & Predecessor \\
\hline 1 & 21 & A & - \\
\hline 2 & 68 & A & - \\
\hline 10 & 185 & A & - \\
\hline 14 & 201 & A & - \\
\hline 5 & 145 & A & - \\
\hline 19 & 86 & A & - \\
\hline 3 & 197 & B & 2 \\
\hline 6 & 71 & B & 5 \\
\hline 20 & 1.2 & B & 19 \\
\hline 4 & 73 & C & 3 \\
\hline 9 & 85 & C & 3 \\
\hline 7 & 151 & C & 6 \\
\hline 21 & 257 & C & 20 \\
\hline 8 & 74 & D & 3,4 \\
\hline 11 & 149 & D & 2,9 \\
\hline 15 & 150 & D & 7,14 \\
\hline 22 & 93 & D & 21 \\
\hline 23 & 187 & D & 20,21 \\
\hline 12 & 61 & E & 11 \\
\hline 24 & 269 & E & 22,23 \\
\hline 13 & 128 & F & 10,12 \\
\hline 26 & 152 & F & 24 \\
\hline 25 & 76 & F & 24 \\
\hline 27 & 70 & F & 24 \\
\hline 16 & 300 & G & 12,13 \\
\hline 29 & 69 & G & 20,26 \\
\hline 28 & 96 & G & 27,25 \\
\hline 17 & 165 & H & 15,16 \\
\hline 30 & 77 & H & 29 \\
\hline 18 & 61 & I & 10,17 \\
\hline 31 & 78 & I & 28,30 \\
\hline 32 & 61 & J & 31 \\
\hline 33 & 96 & K & 32 \\
\hline 34 & 125 & L & 18,30 \\
\hline 36 & 95 & M & 34 \\
\hline 90 & N & 35 \\
\hline W & 241 & O & 35,36 \\
\hline
\end{tabular}

Table 8: Work element arranged according to time values for KWM. 
Citation: E.J. Panggabean, M.R.B. Barjaman and.P.A. Nugraha, 2018. Assembly Line Balancing Using Heuristic Methods. Advances in Natural and Applied Sciences., 12(8): 1-8.DOI: $10.22587 /$ anas.2018.12.8.1

\begin{tabular}{|c|c|c|c|c|c|}
\hline No.Operation & Time (sec) & Column & Predecessor & Station no & Station Time \\
\hline 1 & 21 & A & - & \multirow{10}{*}{ I } & \multirow{10}{*}{$\begin{array}{c}1048.2 \mathrm{sec} \\
(17.28 \mathrm{~min})\end{array}$} \\
\hline 2 & 68 & A & - & & \\
\hline 10 & 185 & A & - & & \\
\hline 14 & 201 & A & - & & \\
\hline 5 & 145 & A & - & & \\
\hline 19 & 86 & A & - & & \\
\hline 3 & 197 & B & 2 & & \\
\hline 6 & 71 & B & 5 & & \\
\hline 20 & 1.2 & B & 19 & & \\
\hline 4 & 73 & $\mathrm{C}$ & 3 & & \\
\hline 9 & 85 & $\mathrm{C}$ & 3 & \multirow{8}{*}{ II } & \multirow{8}{*}{$\begin{array}{c}1146 \mathrm{sec} \\
(19.06 \mathrm{~min})\end{array}$} \\
\hline 7 & 151 & $\mathrm{C}$ & 6 & & \\
\hline 21 & 257 & $\mathrm{C}$ & 20 & & \\
\hline 8 & 74 & $\mathrm{D}$ & 3,4 & & \\
\hline 11 & 149 & $\mathrm{D}$ & 2,9 & & \\
\hline 15 & 150 & $\mathrm{D}$ & 7,14 & & \\
\hline 22 & 93 & $\mathrm{D}$ & 21 & & \\
\hline 23 & 187 & $\mathrm{D}$ & 20,21 & & \\
\hline 12 & 61 & $\mathrm{E}$ & 11 & \multirow{8}{*}{ III } & \multirow{8}{*}{$\begin{array}{c}1125 \mathrm{sec} \\
(18.45 \mathrm{~min})\end{array}$} \\
\hline 24 & 269 & $\mathrm{E}$ & 22,23 & & \\
\hline 13 & 128 & F & 10,12 & & \\
\hline 26 & 152 & $F$ & 24 & & \\
\hline 25 & 76 & $\mathrm{~F}$ & 24 & & \\
\hline 27 & 70 & F & 24 & & \\
\hline 16 & 300 & G & 12,13 & & \\
\hline 29 & 69 & G & 20,26 & & \\
\hline 28 & 96 & $\mathrm{G}$ & 27,25 & \multirow{11}{*}{ IV } & \multirow{11}{*}{$\begin{array}{c}1185 \mathrm{sec} \\
(19.45 \mathrm{~min})\end{array}$} \\
\hline 17 & 165 & $\mathrm{H}$ & 15,16 & & \\
\hline 30 & 77 & $\mathrm{H}$ & 29 & & \\
\hline 18 & 61 & $\mathrm{I}$ & 10,17 & & \\
\hline 31 & 78 & $\mathrm{I}$ & 28,30 & & \\
\hline 32 & 61 & $J$ & 31 & & \\
\hline 33 & 96 & K & 32 & & \\
\hline 34 & 125 & $\mathrm{~L}$ & 18,30 & & \\
\hline 35 & 95 & $\mathrm{M}$ & 34 & & \\
\hline 36 & 90 & $\mathrm{~N}$ & 35 & & \\
\hline 37 & 241 & $\mathrm{O}$ & 35,36 & & \\
\hline
\end{tabular}

$$
\begin{aligned}
L E \text { (Line efficiency) } & =\frac{\sum S T}{\sum S T \max } \times 100 \% \\
S I \text { (Smoothing Index }) & =\sqrt{\sum(S T \max -S T)^{2}}
\end{aligned}
$$

Line Efficiency (LE)

$=(74.24 \mathrm{~min} / 77.80) * 100 \%=\mathbf{9 5 , 4 2} \%$

Smoothing Index $(\mathrm{SI})=\sqrt{ } 335.49=\mathbf{2 . 4 2}$

Balance Delay $=100 \%-\mathrm{LE}=\mathbf{4 . 5 8} \%$

\section{Ranked Positional Weight (RPW) Method:}

Ranked Positional Weight method used and calculated for each element. The method accounted for Time value and its position in the precedence diagram. The RPW is calculated by summing Time and the other times for elements that follow Time in the arrow chain of the precedence diagram Table 9 . After that, rearrange the values of time by using the previous steps, work elements are listed according to RPW value in Table 10.

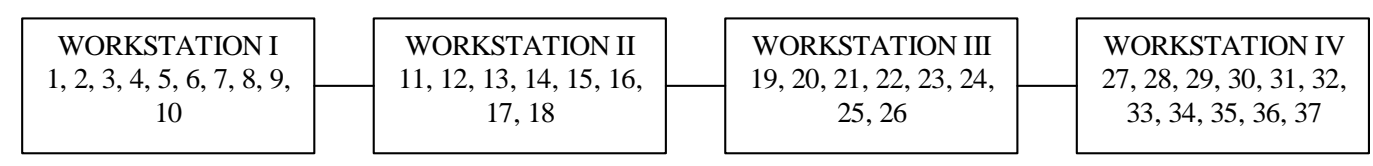

Fig. 4: Configuration Layout for Assembly Line Following LCR 
Citation: E.J. Panggabean, M.R.B. Barjaman and.P.A. Nugraha, 2018. Assembly Line Balancing Using Heuristic Methods. Advances in Natural and Applied Sciences., 12(8): 1-8.DOI: 10.22587/anas.2018.12.8.1

Table 9:Work elements arranged according to RPW.

\begin{tabular}{|c|c|c|c|}
\hline No.Operation & Time (sec) & $\begin{array}{c}\text { RPW (sec) } \\
\text { (Descending) }\end{array}$ & Predecessor \\
\hline 1 & 21 & 4504 & - \\
\hline 2 & 68 & 4483 & - \\
\hline 3 & 197 & 4415 & 2 \\
\hline 4 & 73 & 4218 & 3 \\
\hline 5 & 145 & 4145 & - \\
\hline 6 & 71 & 4000 & 5 \\
\hline 7 & 151 & 3929 & 6 \\
\hline 8 & 74 & 3778 & 3,4 \\
\hline 9 & 85 & 3704 & 3 \\
\hline 10 & 185 & 3619 & - \\
\hline 11 & 149 & 3434 & 2,9 \\
\hline 12 & 61 & 3285 & 11 \\
\hline 13 & 128 & 3224 & 10,12 \\
\hline 14 & 201 & 3096 & - \\
\hline 15 & 150 & 2895 & 7,14 \\
\hline 16 & 300 & 2745 & 12,13 \\
\hline 17 & 165 & 2445 & 15,16 \\
\hline 18 & 61 & 2280 & 10,17 \\
\hline 19 & 86 & 2219 & - \\
\hline 20 & 1.2 & 2133 & 19 \\
\hline 21 & 257 & 2132 & 20 \\
\hline 22 & 93 & 1875 & 21 \\
\hline 23 & 187 & 1782 & 20,21 \\
\hline 24 & 269 & 1595 & 22,23 \\
\hline 25 & 76 & 1326 & 24 \\
\hline 26 & 152 & 1250 & 24 \\
\hline 27 & 70 & 1098 & 24 \\
\hline 28 & 96 & 1028 & 27,25 \\
\hline 29 & 69 & 932 & 20,26 \\
\hline 30 & 77 & 863 & 29 \\
\hline 31 & 78 & 786 & 28,30 \\
\hline 32 & 61 & 708 & 31 \\
\hline 33 & 96 & 647 & 32 \\
\hline 34 & 125 & 551 & 18,30 \\
\hline 35 & 95 & 426 & 34 \\
\hline 36 & 90 & 331 & 35 \\
\hline 37 & 241 & 241 & 35,36 \\
\hline
\end{tabular}

$\begin{aligned} & L E \text { (Line efficiency) }=\frac{\sum S T}{\sum S T \max } \times 100 \% \\ & S I(\text { Smoothing Index })=\sqrt{\sum(S T \max -S T)^{2}} \\ &\end{aligned}$

Table 10: Work elements arranged according to RPW.

\begin{tabular}{|c|c|c|c|c|c|}
\hline No.Operation & Time (sec) & $\begin{array}{c}\text { RPW (sec) } \\
\text { (Descending) }\end{array}$ & Predecessor & Station no & Station Time \\
\hline 1 & 21 & 4504 & - & \multirow{10}{*}{ I } & \multirow{10}{*}{$\begin{array}{c}1070 \mathrm{sec} \\
(17.50 \mathrm{~min})\end{array}$} \\
\hline 2 & 68 & 4483 & - & & \\
\hline 3 & 197 & 4415 & 2 & & \\
\hline 4 & 73 & 4218 & 3 & & \\
\hline 5 & 145 & 4145 & - & & \\
\hline 6 & 71 & 4000 & 5 & & \\
\hline 7 & 151 & 3929 & 6 & & \\
\hline 8 & 74 & 3778 & 3,4 & & \\
\hline 9 & 85 & 3704 & 3 & & \\
\hline 10 & 185 & 3619 & - & & \\
\hline 11 & 149 & 3434 & 2,9 & \multirow{8}{*}{ II } & \multirow{8}{*}{$\begin{array}{c}1215 \mathrm{sec} \\
(20.15 \mathrm{~min})\end{array}$} \\
\hline 12 & 61 & 3285 & 11 & & \\
\hline 13 & 128 & 3224 & 10,12 & & \\
\hline 14 & 201 & 3096 & - & & \\
\hline 15 & 150 & 2895 & 7,14 & & \\
\hline 16 & 300 & 2745 & 12,13 & & \\
\hline 17 & 165 & 2445 & 15,16 & & \\
\hline 18 & 61 & 2280 & 10,17 & & \\
\hline 19 & 86 & 2219 & - & \multirow{8}{*}{ III } & \multirow{8}{*}{$\begin{array}{c}1121.2 \mathrm{sec} \\
(18.41 \mathrm{~min})\end{array}$} \\
\hline 20 & 1.2 & 2133 & 19 & & \\
\hline 21 & 257 & 2132 & 20 & & \\
\hline 22 & 93 & 1875 & 21 & & \\
\hline 23 & 187 & 1782 & 20,21 & & \\
\hline 24 & 269 & 1595 & 22,23 & & \\
\hline 25 & 76 & 1326 & 24 & & \\
\hline 26 & 152 & 1250 & 24 & & \\
\hline 27 & 70 & 1098 & 24 & \multirow{11}{*}{ IV } & \multirow{11}{*}{$\begin{array}{c}1098 \mathrm{sec} \\
(18.18 \mathrm{~min})\end{array}$} \\
\hline 28 & 96 & 1028 & 27,25 & & \\
\hline 29 & 69 & 932 & 20,26 & & \\
\hline 30 & 77 & 863 & 29 & & \\
\hline 31 & 78 & 786 & 28,30 & & \\
\hline 32 & 61 & 708 & 31 & & \\
\hline 33 & 96 & 647 & 32 & & \\
\hline 34 & 125 & 551 & 18,30 & & \\
\hline 35 & 95 & 426 & 34 & & \\
\hline 36 & 90 & 331 & 35 & & \\
\hline 37 & 241 & 241 & 35,36 & & \\
\hline
\end{tabular}

Conclusion:

The goals of this research are balancing the workload allocated to each workstation so that each workstation is completed at a balanced time and increase efficiency or productivity. This research is applying the three heuristic methods to learn about the process of assembly line in Final Area of BMW F30 Series 3 in order to prevent bottleneck (an operation that limits output and production frequency) and keep the assembly crossing smoothly and continuously.

After calculating the existing assembly line the results show the Line Efficiency (LE) is 78,21\%, then heuristic methods (LCR, KWM, RPW) give more efficient assignments/tasks of work element with improvement of Line efficiency which are 95.42\% by using LCR and KWM method and 92.11\% using RPW method. The smoothness index reduced from 18.31 to 2.47 (LCR and KWM) and 3.73 by RPW method. So any one method from above three methods is applicable for balancing of existing assembly line since they show a very slight difference results. 
Citation: E.J. Panggabean, M.R.B. Barjaman and.P.A. Nugraha, 2018. Assembly Line Balancing Using Heuristic Methods. Advances in Natural and Applied Sciences., 12(8): 1-8.DOI: 10.22587/anas.2018.12.8.1

Table 11:Work element arranged according to RPW

\begin{tabular}{|c|c|c|c|c|c|}
\hline No.Operation & Time (sec) & $\begin{array}{c}\text { RPW (sec) } \\
\text { (Descending) }\end{array}$ & Predecessor & Station no & Station Time \\
\hline 1 & 21 & 4504 & - & \multirow{10}{*}{ I } & \multirow{10}{*}{$\begin{array}{c}1070 \mathrm{sec} \\
(17.50 \mathrm{~min})\end{array}$} \\
\hline 2 & 68 & 4483 & - & & \\
\hline 3 & 197 & 4415 & 2 & & \\
\hline 4 & 73 & 4218 & 3 & & \\
\hline 5 & 145 & 4145 & - & & \\
\hline 6 & 71 & 4000 & 5 & & \\
\hline 7 & 151 & 3929 & 6 & & \\
\hline 8 & 74 & 3778 & 3,4 & & \\
\hline 9 & 85 & 3704 & 3 & & \\
\hline 10 & 185 & 3619 & - & & \\
\hline 11 & 149 & 3434 & 2,9 & \multirow{8}{*}{ II } & \multirow{8}{*}{$\begin{array}{c}1215 \mathrm{sec} \\
(20.15 \mathrm{~min})\end{array}$} \\
\hline 12 & 61 & 3285 & 11 & & \\
\hline 13 & 128 & 3224 & 10,12 & & \\
\hline 14 & 201 & 3096 & - & & \\
\hline 15 & 150 & 2895 & 7,14 & & \\
\hline 16 & 300 & 2745 & 12,13 & & \\
\hline 17 & 165 & 2445 & 15,16 & & \\
\hline 18 & 61 & 2280 & 10,17 & & \\
\hline 19 & 86 & 2219 & - & \multirow{8}{*}{ III } & \multirow{8}{*}{$\begin{array}{c}1121.2 \mathrm{sec} \\
(18.41 \mathrm{~min})\end{array}$} \\
\hline 20 & 1.2 & 2133 & 19 & & \\
\hline 21 & 257 & 2132 & 20 & & \\
\hline 22 & 93 & 1875 & 21 & & \\
\hline 23 & 187 & 1782 & 20,21 & & \\
\hline 24 & 269 & 1595 & 22,23 & & \\
\hline 25 & 76 & 1326 & 24 & & \\
\hline 26 & 152 & 1250 & 24 & & \\
\hline 27 & 70 & 1098 & 24 & \multirow{11}{*}{ IV } & \multirow{11}{*}{$\begin{array}{c}1098 \mathrm{sec} \\
(18.18 \mathrm{~min})\end{array}$} \\
\hline 28 & 96 & 1028 & 27,25 & & \\
\hline 29 & 69 & 932 & 20,26 & & \\
\hline 30 & 77 & 863 & 29 & & \\
\hline 31 & 78 & 786 & 28,30 & & \\
\hline 32 & 61 & 708 & 31 & & \\
\hline 33 & 96 & 647 & 32 & & \\
\hline 34 & 125 & 551 & 18,30 & & \\
\hline 35 & 95 & 426 & 34 & & \\
\hline 36 & 90 & 331 & 35 & & \\
\hline 37 & 241 & 241 & 35,36 & & \\
\hline
\end{tabular}

Line Efficiency (LE)

$=(74.24 \mathrm{~min} / 80.60) * 100 \%=\mathbf{9 2 , 1 1} \%$

Smoothing Index $(\mathrm{SI})=\sqrt{ } 335.49=\mathbf{3 . 7 3}$

Balance Delay $=100 \%-\mathrm{LE}=\mathbf{7 . 8 9} \%$

Table 12: Comparison of result obtained from three Methods.

\begin{tabular}{|c|c|c|c|c|c|}
\hline No & Parameter & Existing & LCR & KWM & RPW \\
\hline 1 & Cycle Time (min) & 23.69 & 23.69 & 23.69 & 23.69 \\
\hline 2 & Line Efficiency & $78.21 \%$ & $95.42 \%$ & $95.42 \%$ & $92.11 \%$ \\
\hline 3 & Smoothness Index & 18.31 & 2.47 & 2.47 & 3.73 \\
\hline 4 & Balance Delay & $21.79 \%$ & $4.58 \%$ & $4.58 \%$ & $7.89 \%$ \\
\hline 5 & No of Workstation & 5 & 4 & 4 & 4 \\
\hline
\end{tabular}

\section{REFERENCES}

[1] Nils Boysen, Malte Fliedner and Armin Scholl, 2007. “A classification of assembly line balancing problems” European Journal of Operational Research, 183: 674-693.

[2] Gasperz, Vincent, 2004. Production Planning And Inventory Control. Jakarta: PT Gramedia Pustaka Utama.

[3] Baroto, Teguh, 2002. Production Planning And Inventory Control. Jakarta: Ghalia Indonesia.

[4] Pachghare, Vrittika, 2014. "Assembly Line Balancing Methods-A Case Study" International Journal of Science and Research (IJSR), 3(5).

[5] Nahmias, Steven, 2009. Production and Operations Analysis 6th edition. New York: McGraw-Hill/Irwin.

[6] Seamus M. McGovern, Surendra M. Gupta, 2007. "A balancing method and genetic algorithm for disassembly line balancing". European Journal of Operational Research, 179: 692-708.

[7] Fonseca, D.J., C.L. Guest, M. Elam, C.L. Karr, 2005. A fuzzy logic approach to assembly line balancing, Mathware \& Soft Computing, $12: 57-74$.

[8] Helgeson, W.P., D.P. Birnie, 1961. Assembly line balancing using the ranked posi-tional weight technique. J. Ind. Eng. 